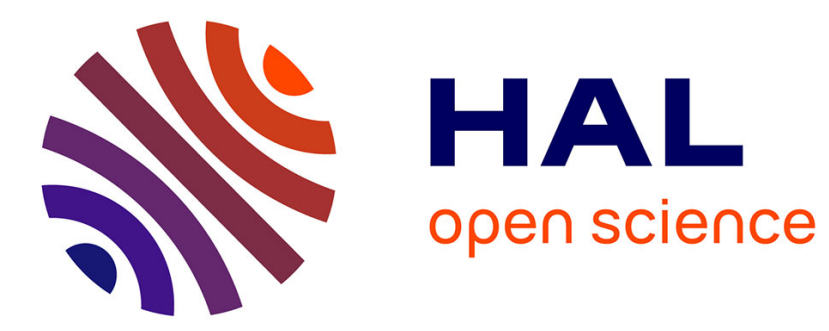

\title{
La frontière au-delà des idées reçues
}

\author{
Anne-Laure Amilhat Szary
}

\section{To cite this version:}

Anne-Laure Amilhat Szary. La frontière au-delà des idées reçues. La revue internationale et stratégique, 2016, État, nation, mondialisation, 102, pp.147-153. 10.3917/ris.102.0147 . halshs$01702807 \mathrm{v} 2$

\section{HAL Id: halshs-01702807 \\ https://shs.hal.science/halshs-01702807v2}

Submitted on 16 Feb 2018

HAL is a multi-disciplinary open access archive for the deposit and dissemination of scientific research documents, whether they are published or not. The documents may come from teaching and research institutions in France or abroad, or from public or private research centers.
L'archive ouverte pluridisciplinaire HAL, est destinée au dépôt et à la diffusion de documents scientifiques de niveau recherche, publiés ou non, émanant des établissements d'enseignement et de recherche français ou étrangers, des laboratoires publics ou privés. 


\section{La frontière au-delà des idées reçues}

Article paru dans La revue internationale et stratégique, Paris : A. Colin : Institut des relations internationales et stratégiques, 2016, pp.147-153. http://www.iris-

france.org/publications/ris-n102-ete-2016/

\section{Anne-Laure Amilhat Szary}

Professeure à l’Université de Grenoble-Alpes / Laboratoire Pacte. Auteure de Qu'est-ce qu'une frontière aujourd'hui ? (Presses universitaire de France, 2015).

Portées au devant de la scène médiatique en 2015, les frontières constituent des objets géopolitiques paradoxaux dont tout un chacun pense pouvoir donner une définition simple. Qui sait pourtant que la plus longue frontière de notre pays est celle qu'il partage avec le Brésil $(730 \mathrm{~km})$ ? Il importe donc de remettre en cause les idées reçues sur la frontière, afin d'appréhender quelques clés permettant de comprendre en quoi ces limites internationales sont devenues des éléments essentiels de la redéfinition des rapports politiques contemporains, et ce, à toutes les échelles. Perçues comme des lignes de démarcation, les frontières sont en effet en train de devenir des dispositifs complexes de tri des flux de la mondialisation plutôt que des limites binaires entre le dedans et le dehors de la nation et / ou de l’État.

Le plus souvent, on part de l'idée qu'il existe des "frontières naturelles », et que les conflits naissent là où l'homme est intervenu pour en modifier le tracé de façon usurpatrice. Cette façon de naturaliser les limites repose sur une perception temporelle souvent erronée du fait frontalier: opposer les frontières des différents continents en fonction de leur date de formation revient à effacer le fait que, même en Europe, elles constituent une réalité historique plus récente qu'on ne l'imagine souvent. Historiciser le fait frontalier permet, dès lors, d'en proposer une approche plus globale et de comprendre ce qui lie aujourd'hui toutes les frontières du monde dans des problématiques comparables, sans perdre de vue que l'idée de frontière comporte des dimensions anthropologiques qui, souvent, nous amènent à une lecture anachronique du passé.

Cette perception essentialisée des frontières se trouve renforcée par leur représentation cartographique comme autant de lignes sur les planisphères auxquels nous sommes familiarisés depuis l'enfance. Elles semblent alors immuables, ou presque. Pourtant, les limites internationales sont bien plus mobiles que la chronologie des soubresauts géopolitiques ne semble l'indiquer : une frontière ne bouge pas seulement à l'occasion d'une guerre, d'une sécession ; même les murs, composés de panneaux en béton amovibles, peuvent voir leur tracé renégocié rapidement. Plus généralement, la diffusion de technologies numériques pour mettre en œuvre le tri des circulations d'hommes et de marchandises, qui constitue la raison d'être des frontières, ne s’opère plus désormais uniquement sur les tracés frontaliers : les points de contrôle sont situés au sein des infrastructures de transport et, plus généralement, dans l’interface de bases de données gérées à distance des lignes. 
Cette évolution nous impose peu à peu une expérience très distincte de la frontière : traditionnellement distante de la plupart des hommes du fait de leur position territoriale périphérique de la majorité des États, la frontière devient pour tous une réalité presque quotidienne. Nous entrons dans une ère où nous pouvons tous les jours faire l'expérience individuelle de la frontière, où celle-ci devient une réalité sociale plus proche que nous ne le croyons.

\section{Une réalité historique plus récente qu’on ne l’imagine}

Bien sûr quand, dès l'Antiquité romaine, Remus franchit le sillon tracé par Romulus autour de la ville que ce dernier projette de fonder, déclenchant chez lui une colère ontologique telle qu'il se jette sur son jumeau et le tue, on voit le mécanisme de la puissance des limites linéaires à l'œuvre et on en conclut souvent que les frontières ont existé de tout temps. Si la distinction matérielle des territoires date effectivement de l'époque de la sédentarisation agricole, et si les premiers murs ont bien été construits soit autour des champs, soit autour des villes, la notion de frontière politique est en fait bien plus récente. Celle-ci date, en effet, des débuts de l'époque moderne, marqués par une transition depuis des formes d'allégeances interpersonnelles féodales vers une conception territoriale de la souveraineté : le pouvoir devient équivalent du contrôle de territoires qui doivent désormais être contigus les uns aux autres. Il est courant de considérer que l'idée de frontière naît à la fin de la guerre de Trente Ans, notamment avec la signature des traités de Westphalie en 1648 : les ambassadeurs des rois d'Europe y négocient alors un principe de stabilité territoriale. Celle-ci ouvre la possibilité d'existence d'États-nations qui vont, dès lors, se consolider selon un principe d'équivalence entre État, territoire et frontières, l'un ne pouvant plus se définir dans l'autre dans une forme de tautologie qui perdure jusqu’à aujourd’hui.

La force de cette «invention » des frontières dans leur modalité européenne moderne repose sur le pouvoir des technologies mises en œuvre pour leur donner vie, et tout particulièrement la cartographie. Inventées à la même époque que les frontières - dans le siècle qui suit la paix de Westphalie -, les cartes topographiques fournissent les supports idoines pour tracer les limites qui sont discutées entre les États. La précision de l'époque - de l’ordre du 1:80 000 met en avant les fleuves, d'une part, les montagnes étant représentées par des chaînons reliés qui en donnent une vision assez linéaire, d'autre part. Cette fabrique à distance de l'objet politique obère bien entendu les modes de vie locaux, cours d'eau et estives constituant traditionnellement dans les espaces ruraux des points de convergence et de partage de la ressource plutôt que des lignes de démarcation.

Cette lecture technique est le plus souvent obérée par le contexte culturel de l'époque : les rois et reines qui se mettent d'accord sur les tracés frontaliers justifient leur pouvoir par une souveraineté de droit divin. Or si le même Dieu qui a créé les fleuves et les montagnes leur a enjoint de régner sur un royaume dont les limites sont naturelles, c'est bien tout le dispositif de pouvoir qui se trouve essentialisé. De façon étonnante, la force de cette notion a largement survécu à la démocratisation de nos sociétés, qui continuent de penser que les frontières naturelles existent quand, en fait, toute frontière, quelle que soit son support, est une construction humaine datée. 


\section{Une réalité spatiale moins fixe que les lignes des cartes}

La puissance de l'imaginaire linéaire dans notre conception de la frontière tient donc aux circonstances historiques de l'invention d'un modèle de frontière européenne, lequel va se diffuser dans le monde avec la colonisation, et s'hybrider avec des traditions différentes dans lesquelles l’idée de limite internationale spatialisée peut être également présente. La question de la ligne reste aujourd'hui omniprésente dans les représentations de la frontière, même dans les travaux artistiques qui cherchent à en détourner le sens : en 2005, quand Francis Alÿs parcourt Jérusalem un pot de peinture verte à la main pour marquer au sol la ligne de l'armistice de 1967 que l'expansion urbaine a effacée du paysage, c'est au pouvoir visuel de la ligne qu'il recourt pour faire émerger le politique à partir du poétique, et inversement ${ }^{1}$.

Ce parcours dans l'espace joue avec la dimension temporelle de la limite internationale: souvent héritée de l'induration d'un rapport de forces, la frontière représente plus qu'un palimpseste, dans la mesure où il s'agit d'un lieu où l'identité est constamment renégociée. Espace-temps en mouvement, elle devient véritablement «mobile » à bien des égards aujourd'hui, au point que le gouvernement italien vient par exemple de faire de la «frontière mobile » un objet de politique publique pour gérer l'évolution du tracé de ses limites alpines localisées sur des lignes de crête glaciaires à la suite de la fonte provoquée par le réchauffement climatique. Les frontières bougent d'abord parce que leur localisation évolue non seulement au gré des soubresauts géopolitiques - création de frontières en Europe à l'effondrement de l'Union soviétique ou de la Yougoslavie, émergence de nouveaux États comme le Soudan du Sud -, mais aussi avec le relief qui lui sert de support - lit des fleuves, glaciers, etc.

Mais la remise en cause de leur fixité est surtout liée aux lieux de mise en application de l'institution frontalière : lieux de régulation et de contrôle, les limites internationales ne sont plus uniquement mise en œuvre en des lieux permanents dédiés - les anciens postes de contrôle -, mais dans une série d'espaces de mobilité à l'intérieur du territoire national contrôle des personnes aux gares et aéroports, contrôle des marchandises sur les voies de circulation qu'elles empruntent. Ces derniers peuvent, de surcroît, ne pas se tenir sur le territoire national, comme en témoigne la politique européenne de visas, qui s’applique dans les bureaux consulaires des pays de départ, notamment africains, ainsi que dans leurs administrations propres, par l'intermédiaire d'un réseau européen d’officiers de liaison «Immigration », institué en 2004. Pour ce qui concerne les marchandises, les vérifications peuvent également être réalisées en amont, dans une procédure de " pré-dédouanement » - ou pre-clearance : l'initiative des États-Unis en la matière (Container Security Initiative Ports, 2002) leur permet de faire intervenir du personnel fédéral dans 58 ports étrangers, représentant $85 \%$ du trafic de conteneurs à destinations de leur pays.

Derrière ces interventions humaines ponctuelles en un nombre croissant de lieux, on trouve en fait une interconnexion grandissante de réseaux d'informations qui produisent une frontière de nature électronique, c'est-à-dire en calcul permanent. Il s'agit surtout d'un lieu qui se

\footnotetext{
${ }^{1}$ Sa performance s'intitulait : «Sometimes doing something poetic can become political and sometimes doing something political can become poetic ».
} 
structure en fonction d'algorithmes de calculs de risques au sein de bases de données reliées, qui forment donc une frontière pixellisée dans sa forme.

Le recours aux technologies révèle un paradoxe entre dématérialisation et démarcation croissante des frontières. Alors qu'il est pour la première fois possible effectivement, notamment grâce au GPS, de démarquer délimiter précisément toutes les frontières, même dans le domaine maritime - et il s'agit là assurément d'un domaine très actif du droit international -, on assiste sur le terrain à des hésitations entre facilitation du passage dynamiques d'ouverture, sélective le cas échéant - et dynamiques de blocage souvent très spectaculaires - recrudescence rapide de murs, qui restent toutefois largement minoritaires à l'échelle du globe, où $8 \%$ des frontières sont actuellement fermées. Au-delà de cette contradiction apparente entre frontières fermées et frontières ouvertes, il est plus juste de dire que l'on observe aujourd'hui des dynamiques d'ouverture et de fermeture (bordering / debordering) sur toutes les frontières du monde.

\section{Une réalité sociale plus proche de nous que nous ne l’imaginons}

Mobile, la frontière cesse donc d’être une réalité périphérique pour la majorité des hommes, tant spatialement que politiquement : point n'est besoin de traverser un pays pour la toucher ; on en fait désormais l'expérience depuis le lieu où l'on vit, même dans des situations géopolitiquement non conflictuelles. Cet usage peut être électronique - frontières des sites Internet comme Netflix ou ITunes n'ouvrant pas les mêmes contenus en fonction des adresses IP -, administrative et économique - demande de papiers d'identité ou de voyage ; conditions de création d'entreprise et d'imposition - ou relationnelle - rapports sociaux et culturels avec des voyageurs, comme avec l'histoire migratoire des familles et des territoires.

Cette interaction est d'autant plus intense qu'elle traverse la matérialité de nos existences. Réalité incorporée, la frontière l'est également à travers le contrôle de nos organismes quand notre identité se trouve de plus en plus définie par des indicateurs biométriques. Les appareils de détection utilisés pour vérifier la sécurité aux frontières, et notamment les nouvelles formes de scanners, déplacent enfin les limites de l'intime en permettant des intrusions visuelles. À cette échelle également, les notions de dedans / dehors apparaissent bousculées.

En tant que sujets globalisés, nous sommes toutes et tous traversés par des frontières nombreuses qui expriment aussi le faisceau de nos identités. Cette différenciation de nos capacités à interagir avec les limites internationales définit une forme de capacité, ou "frontiérité », qui n’est pas nécessairement équivalente à notre niveau de vie : certaines personnes voyagent à la surface du globe sans sortir d'un réseau de lieux standardisés et leur frontiérité optimale en apparence peut être nuancée par leurs difficultés à s'adapter à des contextes spécifiques. Pour autant, la frontière joue de plus en plus un rôle de différenciation territoriale et sociale : en fonction du lieu où l'on naît et du passeport auquel il nous donne accès. Les types de nationalité dessinent, en effet, un monde à géométrie très variable : les uns peuvent, sans visa ou presque, avoir accès à 173 pays - soient 4,3 milliards de leurs semblables sur 73 millions de kilomètres carrés - quand les autres sont restreints à 230 millions de personnes sur seulement 5 millions de kilomètres carrés - dans 28 pays seulement. 
Les limites internationales fonctionnent également comme des lieux d'exacerbation des vulnérabilités : à la frontière, les corps sont, de plus en plus, des corpses, c'est-à-dire des cadavres selon le vocable anglais. Même s’il est rare de mourir précisément sur la ligne, comme dans la série télévisée The Bridge, où un corps est retrouvé sur un pont, minutieusement positionné sur la frontière - l’original a été créé entre la Suède et le Danemark, sur le pont reliant Malmö et Copenhague, mais l'histoire a pu être transposée à Ciudad Juarez / El Paso, entre États-Unis et Mexique, puis dans le tunnel sous la Manche, entre le Royaume-Uni et la France. Les équipes de police qui arrivent sur la scène du crime découvrent rapidement qu'il s'agit en fait de deux morceaux de cadavres de deux femmes raboutés, aux statuts (nationalité, position sociale, couleur de peau, etc.) que tout semble opposer, et donc référer aux deux côtés de la frontière que l'intrigue relie ensuite. La récente crise des naufrages en Méditerranée a rendu cette réalité présente à l'esprit de tous sans que le lien soit généralement assumé entre ces noyades et nos vies. L’évolution récente des frontières nous oblige, en effet, à redéfinir notre rapport à l'altérité de façons qui perturbent notre entendement de la proximité et de la distance.

La dernière évolution - et non des moindres - de la notion frontière concerne le domaine juridique. De façon traditionnelle depuis l'époque moderne, le territoire enclos par des limites internationales était considéré comme un espace d'uniformisation des droits. Base de la souveraineté, le territoire de l’État-nation est convoqué comme justification de la citoyenneté ; c’est le statut géographique qui détermine la condition juridique. Les évolutions récentes dans la gestion des mobilités - restriction de l'asile et criminalisation de la mobilité non justifiée - font apparaître une multiplication de décisions d'exception. Celles-ci sont légales, mais gérées de façon extrêmement différenciée en fonction des guichets. Le résultat géographique de la multiplication de zones d'attentes et de rétention est de provoquer le blocage à la frontière d'une part - certaines personnes ne peuvent pas traverser, leur vie se trouve bloquée dans la limite qu'elles habitent désormais pour une durée extensive - et l'existence de frontière sans « autre côté » d'autre part - impossible de renvoyer quelque part quelqu'un dont on ne sait pas d'où il / elle provient. Tout se passe comme si le statut de la personne, tant juridique, économique ou culturel, faisait désormais la frontière.

La frontière est le lieu du malentendu : il est difficile de se mettre vraiment d'accord sur ce qu'elle veut dire et sans doute est-elle l'un des objets qui permet le mieux de dire la vanité des efforts de traduction. Est-ce un désavantage ? Sans doute beaucoup moins qu'on voudrait nous le faire penser, car la frontière est aussi l'espace de la métaphore par excellence. Il ne s'agit donc pas de trancher sur ce qu'est ou n'est pas une frontière contemporaine, mais bien d'établir combien la compréhension des limites internationales dépasse désormais la binarité avec lesquelles on avait l'habitude de les saisir, notamment en termes d'ouverture / fermeture. Il faut apprendre à gérer des frontières qui s'ouvrent et se ferment à la fois, et dont la réalité géographique se diffuse sur nos territoires à la fois en deçà et au-delà des lignes qui les matérialisent sur les cartes.

Espaces de protection sous l'égide d'un État responsable de la sécurité de ses citoyens, elles deviennent aujourd'hui des lieux de mise en contact des individus avec la violence de la globalisation. Il nous appartient, dès lors, d'en construire une pensée nuancée, qui ne se 
résume pas à la construction d'une situation de citoyenneté exclusive et considère, au contraire, que le différentiel que met en jeu toute frontière constitue une ressource, non seulement culturelle, mais aussi économique et politique. Ses invariants anthropologiques font que les frontières nous concernent tous : elles constituent donc un levier d'action symbolique d'importance. Donnons-nous les moyens de dépasser les idées reçues pour faire de la frontière un point de départ de débats renouvelés et nuancés pour penser le vivre-ensemble à toutes les échelles. 